\title{
Polysaccharide-Protein Interactions and Their Relevance in Food Colloids
}

\author{
Amit K. Ghosh and Prasun Bandyopadhyay \\ Additional information is available at the end of the chapter
}

http://dx.doi.org/10.5772/50561

\section{Introduction}

Polysaccharides and proteins are natural polymers that are widely used as functional ingredients for various food colloids or emulsion formulations. Majority of food emulsions are constituted with polysaccharide and protein combinations. They are the essential ingredients of any food colloid formulation mainly due to their ability to change product shelf life by varying food texture (Schmidt \& Smith, 1992; Schorsch, Jones \& Norton 1999). Their interaction in the formulation thus finds many applications particularly in new food formulation development. Due to complex formation and creation of nano or micro structures (aggregation and gelation behavior) they generally change the rheological properties of food colloids which may affect the food product texture and colloidal stability (Benichou, 2002; McClements, 2005, 2006, 2007; Dickinson, 2003). Polysaccharide and protein interactions in solution and interfaces have been studied by several groups (Dickinson, 2003, 2008; Bos \&Van Vliet 2001; Carrera \& Rodríguez Patino 2005; Krägel, Derkatch, \& Miller, 2008; Koupantis, \& Kiosseoglou, 2009; Mackie, 2009). However, despite the vast advancement made in the recent past, polysaccharide and protein interactions in food hydrocolloids continue to be one of the most challenging topics to understand.

Proteins, being surface active can play major role in the formation and stabilization of emulsions in the presence of polysaccharide, while interacting through electrostatic or hydrophobic-hydrophobic interactions. On the other hand, polysaccharides being hydrophilic in nature generally remain in aqueous phase thus help in controlling the aqueous phase rheology like thickening, gelling and acting as stabilizing agents. The formation and deformation of polysaccharide-protein complexes and their solubility depend on various factors like charge and nature of biopolymers, $\mathrm{pH}$, ionic strength and temperature of the medium and even the presence of surfactant of the medium (Ghosh \& Bandyopadhyay, 2011). If $\mathrm{pH}$ of the medium is reduced below isoelctric point $(\mathrm{pI})$ of the 
protein present then net positive charge of the protein will become prominent which will interact with negatively charged polysaccharide to form stable electrostatic complex. Similarly, if solution $\mathrm{pH}$ increased more than protein $\mathrm{pI}$, the net negative charge of protein will tend to form complex with positively charged polysaccharides (Xia \& Dubin, 1994; Dickinson, 2008; Turgeon, Schmitt \& Sanchez, 2007). Generally, chances of weaker complex formation is more when solution $\mathrm{pH}$ is almost equal to protein $\mathrm{pI}$, because at that $\mathrm{pH}$ range surface charge of protein becomes nearly zero. However, at very high concentration, similarly charged biopolymers repel each other and the net repulsion make the system unstable (separate as two distinct phases) which is known as thermodynamic incompatibility. Incompatibility in the system occurs at $\mathrm{pH}$ higher than the protein $\mathrm{p} I$ and at higher ionic strength (Grinberg \& Tolstoguzov, 1997). Thus by varying $\mathrm{pH}$ and ionic strength of the medium one can achieve a control on the polysaccharide-protein interactions.

Polysaccharides and proteins both contribute to the structural and textural properties of food by changing rheology of food emulsions through their gelling networking system (Dickinson, 1992). Non-covalent interactions between polysaccharide and protein in any emulsion formulation play a major role to change the interfacial behavior and stability of the food colloids. The driving force for these non-covalent interactions is electrostatic interactions, hydrophobic interactions, H-bonding and Van der Waals interactions. Recent literatures also focus on how protein and polysaccharide molecules can be linked together by covalent bond. At $\mathrm{pH}$ close to protein $\mathrm{pI}$ this Maillard-type conjugates were used to improve the colloidal stability and interfacial structure of proteins in certain conditions (Jiménez-Castańo, Villamiel, \& López-Fandiňo, 2007; Benichou, Aserin, Lutz \& Garti, 2007)). Recent developments in the field describe interfacial physico-chemical properties of polysaccharide-protein mixed systems (Rodríguez Patino \& Pilosof 2011). In this chapter, we would like to focus more on polysaccharide and protein non-covalent interaction studies and their effect towards food colloids stability.

\section{Nature of polysaccharide-protein complex}

Polysaccharide and protein complex formation is mainly driven by various non-covalent interactions, like electrostatic, H-bonding, hydrophobic, and steric interactions (Kruif et al 2001). Protein carries +ve or -ve zeta potential based on the $\mathrm{pH}$ of the medium (+ve at $\mathrm{pH}$ lower than $\mathrm{pI}$ and vice-versa). This +ve or -ve electrical charge on the protein chain point towards the presence of different amino acids in the protein molecules and their mode of ionization at different $\mathrm{pH}$ ranges (Fig. 1). Carboxylate polysaccharides get deprotonated (become anionic) at a $\mathrm{pH}$ range higher than its pKa (Fig. 1). This electrical charge on the back bone of protein or polysaccharide chain is responsible for electrostatic attraction or repulsion between them. Again, presence of $-\mathrm{COOH}$ group on the polysaccharide and $-\mathrm{NH}_{3}$, - $\mathrm{COOH}$ groups on the protein chain are the sources of hydrogen bonding between these two bio-polymers. Extent of both of this hydrogen bonding and electrostatic interaction depends on the solution parameters such as $\mathrm{pH}$, ionic strength, temperature etc. Except these ionic patches on the bio-polymers, few non-polar segments are also present on the bio-polymers, 
which are responsible for the hydrophobic staking with each other. Even though solution parameters are important factors to control the different mode of interactions between protein and polysaccharide, type of proteins/polysaccharides, molecular weight, charge density, and hydrophobicity of the bio-polymers are also play significant role towards the extent of complexation between two bio-polymers at a fixed condition.

$$
\text { At pH }<<\text { PI/pKa }
$$<smiles>[R]C([NH3+])C(=O)O</smiles>

$\&$

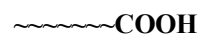

$$
\text { At pH }=\mathbf{P I} / \mathbf{p K a}
$$<smiles>[R]C(N)C(=O)O</smiles>

$\&$
At pH $>>$ PI/pKa

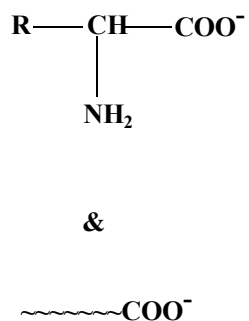

Figure 1. Variation of charge density on the polysaccharide and protein chain at various $\mathrm{pH}$ ranges.

In general, interactions between proteins and polysaccharides are quite explored where large numbers of report have been published based on the interactions between oppositely charged "protein-polysaccharide" systems (Dmitrochenko et al 1989; Bengoechea et al 2011, Stone \& Nickerson 2012). Although electrostatic attraction is the main driving force for the complexation between protein and polysaccharide, but it is also reported that hydrogen bonding and hydrophobic interaction plays a secondary role for stability of the "proteinpolysaccharide" aggregates (McClements, 2006). The extent of hydrogen bonding and hydrophobic interaction also depends on temperature (Weinbreck et al, 2004). In 2009 Nickerson and co-workers(Liu, Low, \& Nickerson, 2009) have reported that pea protein and gum acacia complex stabilize at low temperature due to increase in hydrogen bonding interactions and destabilize at high temperature due to decline in hydrogen bonding interactions. Temperature also plays an important role to decide the protein conformations (folded or unfolded). In 2007, Pal (Mitra, Sinha \& Pal, 2007) and coworkers have reported that human serum albumin unfolds at higher temperature and undergoes in reversible refolding conformations upon cooling (below $60^{\circ} \mathrm{c}$ ). Unfolded conformations of protein expose more reactive sites (amino acids) to the solvent phase, thus more chances of interactions (or binding) with polysaccharide. Binding of anionic polysaccharides ( $\mathrm{pH} \sim \mathrm{pK}$ ) to the cationic proteins (at $\mathrm{pH}<\mathrm{p} I$ ) result both soluble and insoluble complexes (Magnusson \& Nilsson, 2011). Initial binding of polysaccharides (anionic) to the proteins (cationic) cause charge neutralizations, which lead to the formation of insoluble "protein-polysaccharide" aggregates (Schmitt et al, 1998). Further binding of anionic polysaccharides to those neutral aggregates make it effectively anionic, which leads to formation of soluble complexes. But binding of anionic polysaccharides with anionic proteins $(\mathrm{pH}>\mathrm{p} I)$ are also known and governed by the interactions between anionic reactive sites of polysaccharide and small cationic reactive sites of protein (Fig. 2). Binding of anionic polysaccharides to the cationic 
side of proteins (at $\mathrm{pH}>\mathrm{p} I$ ) result in formation of anionic "protein-polysaccharide" aggregates, thus soluble complexes. Therefore, concentration of polysaccharides and $\mathrm{pH}$ play an important role towards the solubility of "protein-polysaccharide" aggregates.

Two bio-polymers can exist either in a single phase systems or in a phase separated systems depending on the nature of bio-polymers, their concentration, and solution conditions. When two bio-polymers carry opposite charge, then either they agglomerates to form soluble complexes (single phase) or insoluble precipitates (2-phase system). On the other hand, when two non-interacting bio-polymers mixed together, either they exist in a single phase system (where two separate entities distributes uniformly throughout the medium) or exist as two distinct phases (each phases comprise different bio-polymer). Therefore, in the protein-polysaccharide system, phase separation occurs through two different mechanisms which are associative phase separation and segregative phase separation (Tolstoguzov, 2006). Associative phase separation is the aggregation between two oppositely charged bio-polymers (electrostatic attraction driven), leads to the phase separation, where one phase is enriched with two different bio-polymers (coacervation or precipitation) (Fig. 3). Segregative phase separation occurs either due to strong electrostatic repulsion (between two similarly charged bio-polymers) or because of very high steric exclusion (between two neutral bio-polymers). In this case, at low concentration, two biopolymers can co-exist in a single phase whereas at higher concentration, it starts phase separation. (Fig. 3).

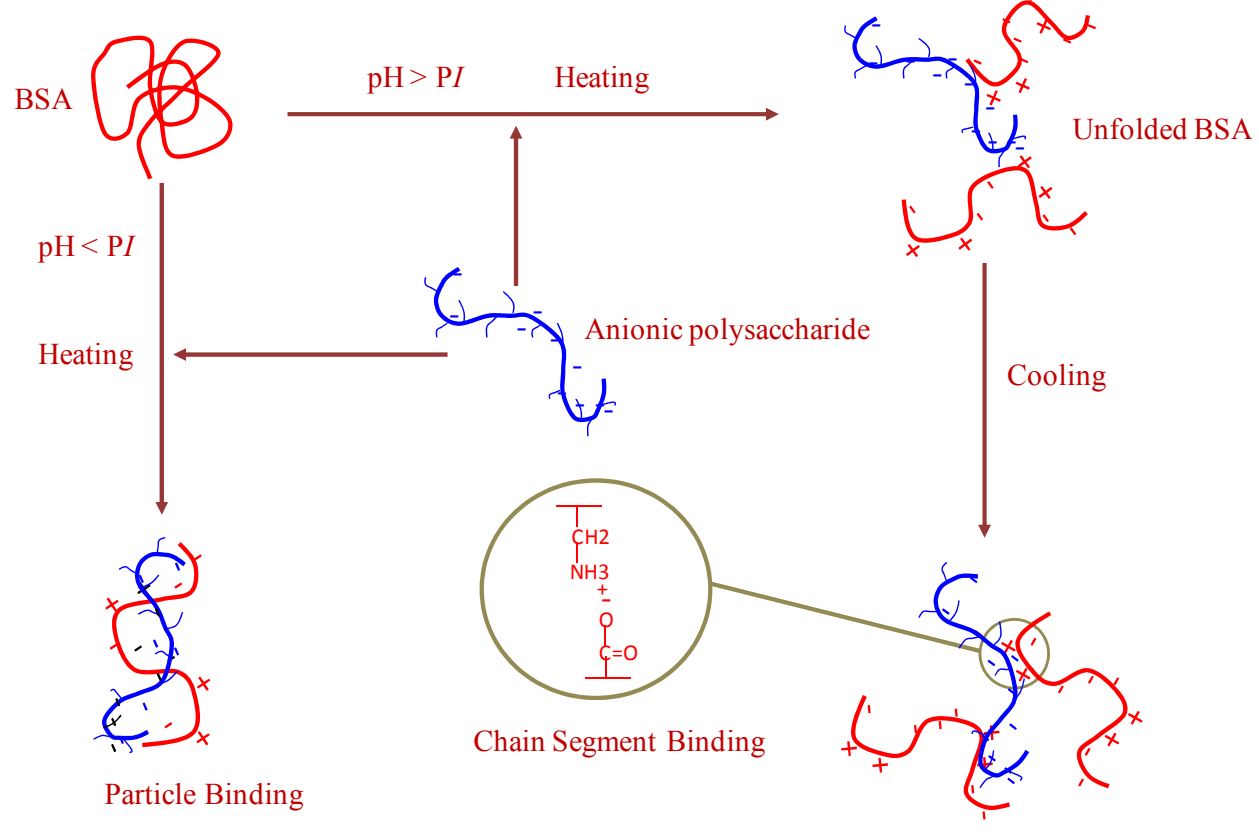

Figure 2. Interaction between polysaccharide and protein at various $\mathrm{pH}$. 


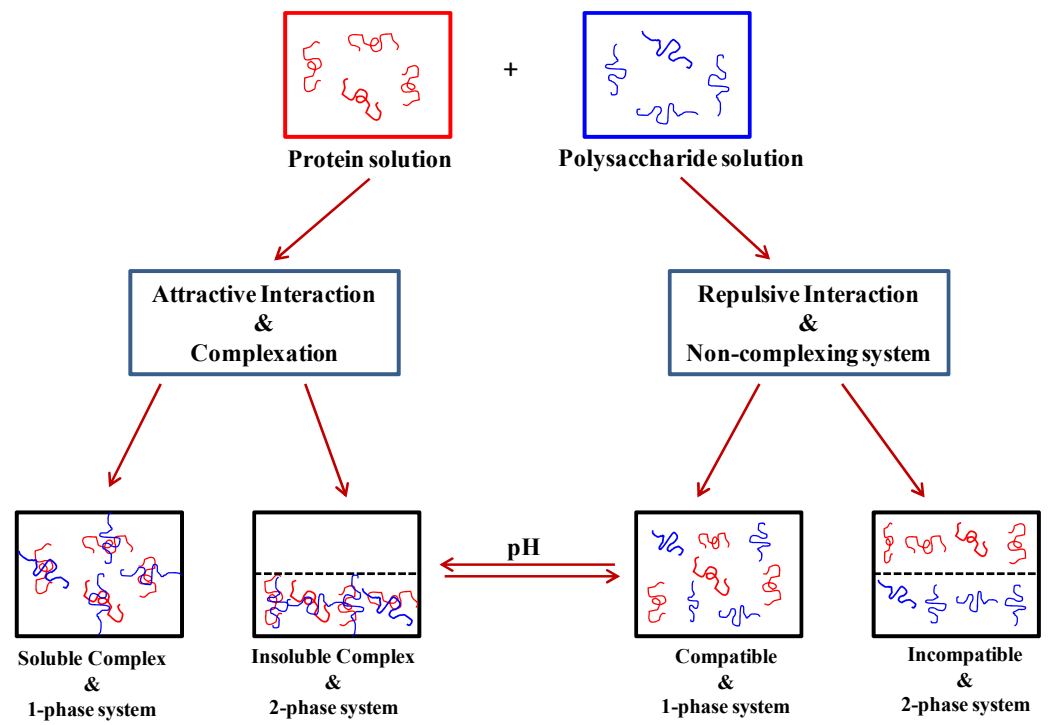

Figure 3. Schematic representation of the possible mode of interaction between polysaccharides and proteins.

\section{Functional properties of polysaccharide-protein complexes related to food application}

Polysaccharide-protein complexes exhibit a wide range of interesting properties, such as surface activity to stabilize air-water or oil-water interfaces, viscosifying, and gelling properties etc. Viscosifying and gelling ability of polysaccharide-protein complexes help to obtain gel-like processed food products without any thermal treatment in the process. The interfacial properties of these complexes help to impart stability into the emulsion food products. Also, protein-polysaccharide complexes are able to encapsulate several active ingredients; hence they act as delivery systems for many bioactives or sensitive molecules in food formulations. These complexes are also known to vary bulk/interfacial structures, textures and shelf-life stability of the food colloids. In the following section we will discuss this polysaccharide-protein interaction in light of their functional properties.

\subsection{Viscosity of polysaccharide-protein complex and air-water foam stability}

Viscosity and gelling are the rheological property which depends on the molecular characteristics of the biopolymers, such as their molecular weight, shape, chain flexibility. Other factors are the concentration, interaction between the biopolymers and water, as well as solution parameters like: $\mathrm{pH}$, ionic strength and presence of other components/ligands etc. Interactions between polysaccharide and protein have been proven to widen the functional properties of each individual biopolymer. Rheological properties of polysaccharide-protein complex lead to new rheological behaviors different from each individual biopolymer. 
Association of two bio-polymers is expected to increase the bulk viscosity of the system as entities of larger sizes are formed. The rheological behavior of several proteinpolysaccharide mixed systems have been studied and ranged from viscous to viscoelastic properties showing elastic behavior is reported. The hydrated polysaccharide-protein complexes increase viscosity and rheology of the system found to be depends on the nature and structure of polysaccharides. Viscous property of gum acacia-protein coacervates attributes to the globular conformation of the polysaccharide, whereas the same protein with linear pectin results in gel-like system. Beside the nature of the individual bio-polymer solution property and concentration of bio-polymers are also known to affect the rheology of the system (Dickinson, 2011). For example, it was found that $\mathrm{pH}$ played a major role in the viscosity of the coacervate phase. A maximum viscosity was obtained at $\mathrm{pH} 4.0$, where concentration of whey protein and gum arabic in the coacervate phase was maximum and extent of electrostatic attraction was highest. This suggests that the electrostatic interactions between whey protein and gum arabic were responsible for the highly viscous behavior of the coacervates. Whereas, the same composition of whey protein and gum arabic at $\mathrm{pH}$ above protein $\mathrm{pI}$ (i.e. comparatively lower electrostatic interactions) showed more elastic nature than viscous. Ionic strength and protein/polysaccharide ratio is also known to play an important role towards the rheology of polysaccharide-protein systems. For example, optimal salt concentrations $(0.21 \mathrm{M} \mathrm{NaCl})$ favor the coacervation of $\beta$-lactoglobulin with pectin at higher concentration and produce much stronger gel strength. For better gelling property, it is necessary to control the parameters which required to form coacervate, because strong associative interaction decrease the solubility of complexes and hence lower the hydration capacity of the complex, which leads to decrease in the viscosity (Schmitt \& Turgeon, 2011; Kruif, et al 2004).

Viscoelastic properties of polysaccharide-protein complexes also play an important role towards the foam stability in variety of food products. In case of air-water system foam can be define as the air entrapment by a thin liquid film (water), where this liquid film is stabilized by some surface active molecules. Stability of the foam increases with the increase in the stability of the interfacial liquid film, because lower stability of this interfacial liquid film can lead to the diffusion of air entrapped inside the foam. Viscosity of this liquid film is another parameter by which one can control the diffusion rate of air entrapped inside the foam. Therefore, higher stability and viscosity of the interfacial liquid film leads to lower diffusion of air entrapped inside the foam and increase the foam stability. Schmitt and coworkers have studied the air-water interfacial property of $\beta$-lactoglobulin-acacia gum complexes at $\mathrm{pH} 4.2$ [Schmitt et al 2005]. The group has reported that although surface activity of the complex is similar with the pure protein, but complex forms much stronger viscoelastic interfacial film with thickness of about $250 \AA$. As a result, gas permeability of thin-film stabilized by the complexes was significantly reduced $\left(0.021 \mathrm{~cm} \mathrm{~s}^{-1}\right)$ compared to pure $\beta$-lactoglobulin $\left(0.521 \mathrm{~cm} \mathrm{~s}^{-1}\right)$. This phenomenon suggests that stability of foam (stabilized by protein-polysaccharide complex) is higher compared to the foam stabilized by protein alone. 
The likely explanation of the higher foam stability and different interfacial properties of coacervate is that protein-polysccharide complexes are able to re-organize at the interface by coalescence, forming interfacial microgel. These findings were applied for the ice cream formulation for improved air bubble stability (Schmitt C, Kolodziejczyk E. 2010). Similarly, gelatin has been replaced by whey protein isolate-gum acacia complexes to improve the bubble stability in chilled dairy products (Schmitt C, Kolodziejczyk E. 2010). In case of stabilization by complexes, variation in ratio of biopolymers could be used to control the size of the complexes, hence their surface activity. In addition to that, viscoelastic properties of the air-water interfacial film is possible to tune by either adsorbing two biopolymers simultaneously or by the sequential adsorption of protein followed by polysaccharide. As for example, $\beta$-lactoglobulin-pectin complexes are known to stabilize the air-water interface. In this case, thickness of the film obtained from the sequential adsorption of protein and polysaccharide was higher ( $450 \AA$ ) than the adsorption of complexes (250 $\mathrm{A}$ ) (Ganzelves et al 2008).

In contrary to air-water foam stability, use of polysaccharide-protein complexes for the stabilization of oil-water emulsion (Martínez et al 2007) has received much more attention. Use of these polysaccharide-protein hydrocolloids as an emulsion stabilizer will be discussed in the next section.

\subsection{Oil-water emulsion stability}

Emulsion is a uniform dispersion of liquid droplets within a continuous matrix of a second immiscible liquid, stabilized by surface active molecules. These stabilizers are termed as emulsifier. In the context of the present topic, we will limit our discussion to the role of biopolymers as emulsifier. Generally, emulsifier has the amphiphilic character to adsorb onto the interface of liquid droplets, which can prevent the phase separation of two immiscible liquids. For a fixed emulsifier, stability of the emulsion depends on few factors, such as rate of adsorption of the emulsifier, concentration of emulsifier, etc. At low concentration of emulsifiers, emulsion system fails to retain its initial droplet size. This destabilization can take place through different mechanisms. In case of poor coverage of the interface by liquid droplets, they can coalesce with each other to form a bigger droplet (Fig. 4). Few examples are also reported, where polymer adsorbed onto the interface of liquid droplets thus bridge between two such liquid droplets and initiates bridging flocculation. Interestingly, emulsions at high emulsifier concentration produces stable oil droplets due to better coverage of the interfaces of the liquid droplets ( Liu \& Zhao, 2011).

Emulsion is possible to achieve by using many surface active agents, such as small surfactant molecules, bio-polymers (proteins or polysaccharides, hydrocolloids (proteinpolysaccharide complexes), and inorganic particles. Stability of those emulsion systems mainly governs by the two important factors. First, repulsive force between two closely approaching liquid droplets; second, Ostwald ripening, which involves disappearing of smaller droplets in expense of the formation of larger one. Higher degree of repulsion between the two neighboring droplets results in maximum stability due to least chances 
of coalescence. Repulsive force between the two liquid droplets govern by the inter droplet distance, i.e. thickness of the thin liquid film between two closely approaching droplets. Thickness of this liquid film depends on the space occupied by the adsorbed molecules (emulsifier) at interface of the droplets. Emulsion generally get stabilized by different emulsifiers present in the formulations such as surfactants, proteins, or hydrocolloids (protein-polysaccharide complexes) and the relative thickness of the liquid film between two closely spaced droplets lies in the order of hydrocolloids $(5-10 \mathrm{~nm})>$ proteins $(1-5 \mathrm{~nm})>$ surfactants $(0.5-1 \mathrm{~nm})$ (Fig. 5). Therefore, stability of the emulsion droplets expected to be higher when they are stabilized by protein-polysaccharide complex compared to the same stabilized by protein or surfactant molecules. In addition to the thickness of the liquid film between two closely spaced droplets, rate of desorption of the emulsifiers from the interface is another important factor. Adsorption of emulsifier molecules (like surfactants, proteins etc.) at the liquid interface is highly reversible. Desorption of the emulsifiers from the liquid interface governs the instability of the system. According to this fact, emulsion stabilized by particles (size ranges from $10 \mathrm{~nm}$ to several $\mu \mathrm{m}$ ) is likely to have indefinite stability, because of the maximum thickness of thin liquid film in between two closely spaced droplets and maximum desorption energy of the particles from the liquid interface. Despite of this theoretical consideration, experimental evidence by Tcholakova et al. does not support this hypothesis that particle stabilized emulsion are more stable compared to surfactant or protein or hydrocolloids stabilized emulsion (Tcholakova et al. 2008).

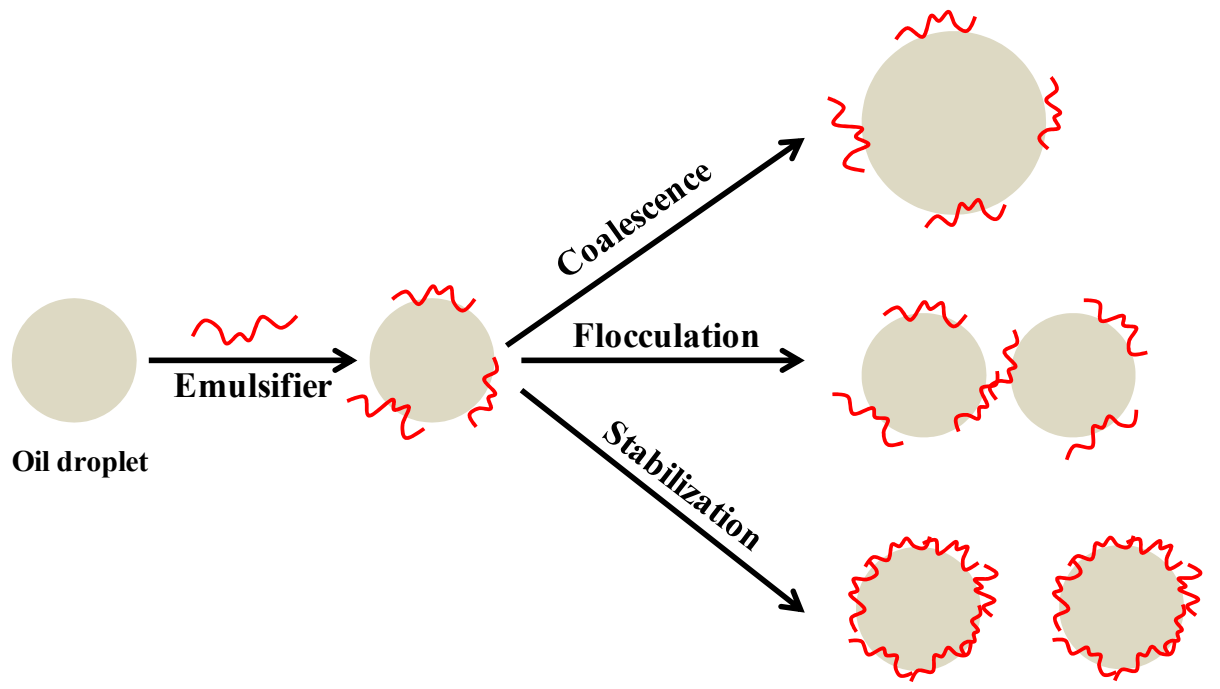

Figure 4. Schematic representation of mode of stabilization and destabilization of oil droplets in an oilwater emulsion. 
A

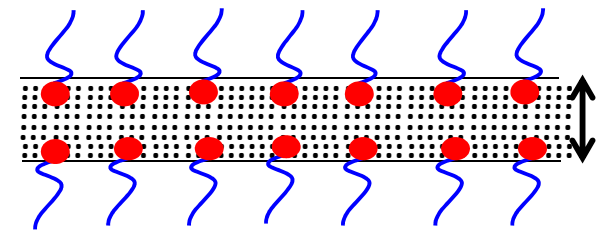

B
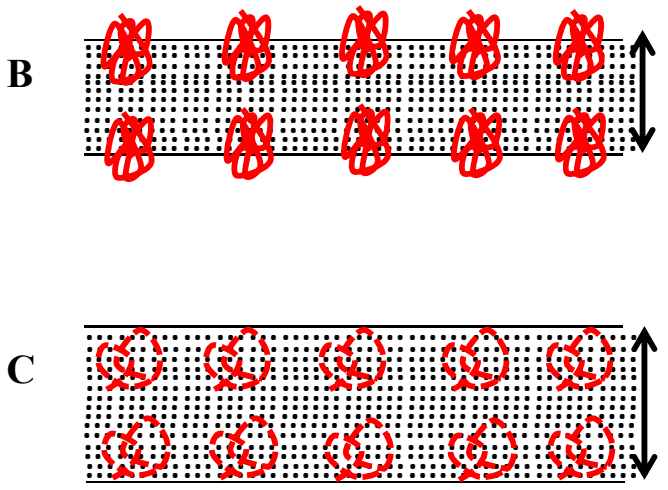

Figure 5. Schematic representation of the relative thickness of the thin liquid film between two closely spaced droplets, stabilized by A) surfactant, B) protein, and C) protein-polysaccharide hydrocolloids.

Another factor guides the emulsion instability is Ostwald ripening, which is disappearance of small size droplets at the expense of the larger droplets formation. Driving force for the Ostwald ripening is the difference in the chemical potential of the smaller and larger droplets. Mass transfer takes place between the droplets by diffusion process. Therefore, Ostwald ripening process requires the solubility of the dispersed phase into the continuous phase to initiate the diffusion process. Type of the emulsifier also plays an important role towards the Ostwald ripening process. Emulsion stabilized by the water soluble surfactant molecules has lower interfacial tension, which reduces the thermodynamic driving force of Ostwald ripening. Adsorption of the surfactant molecules at the droplet interface is a reversible phenomenon. Reversible desorption and adsorption of the surfactant molecules from the interface of the liquid droplet increases the rate of mass transfer between the dispersed droplets, hence increase the Ostwald ripening. The chances of desorption of emulsifier is less in case of the emulsions stabilized by protein molecule because it provides a thicker layer (elastic layer) around the droplets and greater surface coverage of the interfacial area. These factors reduce the ripening process in the protein stabilized emulsion. Ostwald ripening process is possible to avoid completely, only if emulsion is stabilized by insoluble particles (due to very high desorption energy) or thickness of the elastic layer around the dispersed droplets is equal to the droplet radius (Kabalnov, 2001). For this reason particulate emulsions are able to prevent the ripening process completely. Whereas hydrocolloid (protein-polysaccharide complexes) mostly behaves like a soft polymer, more resembles with the protein structures compared to solid particles, which cannot completely avoid the ripening process. 
Protein-polysaccharide complex stabilized emulsions are possible to obtain by using two alternative ways. One of them involves addition of charged polysaccharide solution to a primary emulsion which is already stabilized by the protein as single emulsifier, to produce emulsion droplets having a protein-polysaccharide 'bilayer' surface coating (Fig. 6B). Another method involves addition of an aqueous solution containing the proteinpolysaccharide complexes as an emulsifying agent following homogenization (Fig. 6A). For convenience, the first method is termed as 'bilayer emulsions' and second one is termed as 'mixed emulsions'. The bilayer approach is also commonly known as 'layer-bylayer' approach. Recently, it has attracted significant importance because of its use in nano-encapsulation and protection of emulsions against severe environmental stresses. The major problem lies in 'layer-by-layer' approach, where emulsion droplet tends to flocculate. Flocculation during the 'layer-by-layer' adsorption takes place because of two different mechanisms: a) bridging flocculation, b) depletion flocculation. Bridging flocculation takes place at low polysaccharide concentration when droplet collisions occur faster than the rate of polysaccharide saturation of the protein-coated droplet surfaces. Depletion flocculation occurs at higher polysaccharide concentration when unadsorbed polysaccharide exceeds a critical value. For this reason it is convenient to make emulsions with protein and polysaccharide present together before homogenization compared to 'layer-by-layer' approach. Recently direct comparison between the two techniques has been demonstrated experimentally, which shows that the more convenient mixed emulsion approach leads to better stability behavior than the bi-layer approach(Camino et al 2011).

A

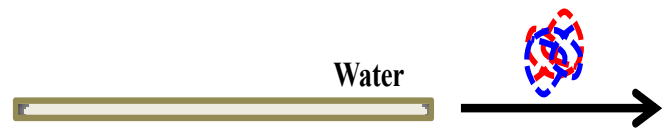

oil

B

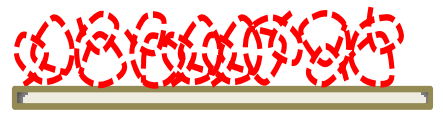

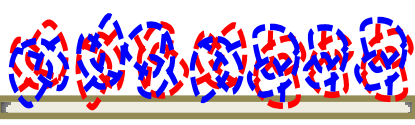

r

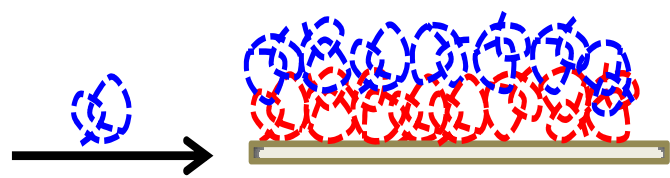

Figure 6. Schematic illustration of two alternative approach of preparing oil-water emulsion using protein-polysaccharide complexes as an emulsifier. A) Mixed emulsions, both protein and polysaccharide present together during emulsification. B) Bilayer emulsion, polysaccharide added to emulsion prior stabilized by protein.

\subsection{Encapulation and release of active molecules}

Generally, encapsulation includes all aspects of protection or stabilization of active molecules (flavors and bio-actives) against several external drastic conditions (such as heat, redox potential, shear, temperature, light, oxygen, moisture, etc.). Controlled release facilitates the delivery of the encapsulated material to the targeted place with the optimal kinetics. Conditions for the encapsulation of active molecules depends on the sensitivity 
(thermal and redox stability) and nature (solubility in oil and water) of the active components but release can be controlled by mechanical process, $\mathrm{pH}$ variations (acidic conditions in the stomach, neutral in the intestine) or enzymatic actions etc. As for example, Peniche et al. has shown the encapsulation of shark liver oil (rich in poly unsaturated fatty acids) using chitosan-alginate system to mask the unpleasant taste of oil. These capsules disrupts by enzymes, like lipase or pancreatine. Initially it was resistant to the acid environment of the stomach, but after 4 hour in the intestinal $\mathrm{pH}$ (pH 7.4), the capsule walls weakened and delivers the active molecules.

Another important application of this aspect is the encapsulation of flavor molecules. Recently, Yeo et al. has shown that gelatin-acacia gum coacervate can encapsulate flavors which can be released during cooking in baked goods (Yeo et al. 2005). Weinbreck et al. (Weinbreck et al. 2004) has shown that Whey protein-acacia gum coacervates can encapsulate lemon and orange flavors and their release under mechanical action like chewing. Encapsulation was one of the first applications of gum arabic-gelatin coacervates (Bungenberg de Jong and Kruyt, 1929). Viscous coacervate was made at a temperature higher than the gel point of gelatin and during cooling, interfacial rigidity increases which lead to a stable gelled shell around the microcapsule. This rigid shell disrupts after consumption, gelatin melts easily in the mouth and therefore releases the encapsulated actives. In addition to gelatin-acacia gum, several other protein-polysaccharide systems have been evolved (whey proteins, plant proteins, pectin, and xanthan gum) to broaden the encapsulation techniques. Beside these polysaccharide-protein combinations, process parameter for encapsulation $(\mathrm{pH}$, ionic strength, macromolecular ratio, and drying/homogenization procedure) also plays an important role to modulate the physical properties (thickness, swelling rate, etc.) of the coacervate layer in the microcapsules (Savary et al 2010). Use of cross-linking agents can further harden the coacervate layer after formation of the microcapsules. As for example, use of trans-glutaminase can introduce covalent linkages between carboxyl group of a glutamine and amino group of lysine in the protein molecules. Alternatively, formaldehydes and glutaraldehydes have also been studied although they are non-food grade reagents. Recently, tannic acid, plant phenolics, citral molecules and glycerin have been studied as food grade alternatives (McClements, 2010).

Contrary to the encapsulation through coacervation, the bi-layer emulsion technique (formed by successive adsorption of biopolymers at the interface) is another way to study the microcapsule properties. Recently, McClements group has described that bi-layer approach of encapsulation has a better control of the interface structure, charge, thickness and permeability with improved stability and controlled release of actives. Group has reviewed this research area and discussed multilayer emulsions in light of bioavailability control and release of actives to the specific site of action depending on layer composition and properties (McClements, 2011). Sagis et al. has used high molecular weight pectins and pre-heated whey proteins (denaturation of protein) for the encapsulation through multilayer approach. (Sagis et al 2011). 


\section{Summary}

Basic understanding of supramolecular chemistry which span among origin and nature of the various non-covalent molecular interactions between polysaccharides and proteins can be widely used to create various desirable nano and macro structures which are quite significant in food colloids/formulations. Food product texture modulation and colloidal stability can be achieved by controlling protein-polysaccharide interactions. Modulation of this interaction by varying medium conditions like $\mathrm{pH}$, ionic strength etc. one can create many possibilities towards rheological properties of food colloids which may affect the emulsion stability. Protein-polysaccharide interactions are well characterized in various $\mathrm{pH}$ conditions. Although, the interaction depends on type and nature of biopolymers, no structure-activity correlation has been established until now. Moving forward, there is a huge demand to establish a correlation between biopolymer structures and interaction efficiency. The creative manipulation of polysaccharide-protein interactions can open up a completely new dimension in health and nutrition platform. Food particle travels from mouth to gut in various $\mathrm{pH}$ environments (for example $\mathrm{pH}$ decreases when it moves from mouth saliva to stomach and increases when partially digested food particle passes from stomach to small intestine), thus one can design a smart polysaccharide-protein complex system which can encapsulate or slow down and trigger or release of nutrients in various stages of digestion process depending on the $\mathrm{pH}$ of the system and any particular health demand. Such kind of $\mathrm{pH}$ sensitive system design and product development which works in vivo is a real challenge for the food scientists. Unfortunately, general understanding of protein-polysaccharide interactions, their bulk and interfacial properties toward emulsion stability is not completely understood and requires more systematic investigation in future to unveil its full potential.

\section{Author details}

Amit K. Ghosh and Prasun Bandyopadhyay*

Unilever $R$ \& D Bangalore, Whitefield, Bangalore, India

\section{References}

Bengoechea, C., Jones, O. G., Guerreo, A., \& McClements, D. J. Food Hydrocolloid, Vol. 25, 2011, pp. 1227-1232

Benichou, A., Aserin, A. \& Garti, N. Journal of Dispersion Science and Technology. Vol. 23, 2002, pp. 93-123

Benichou, A., Aserin, A., Lutz, R. \& Garti, N. Food Hydrocolloid, Vol. 21, 2007, pp. 379-391

Bos, M. A, \& Van Vilet, T. Advances in Colloid \& Interface Science. Vol. 91, 2001, 437-471

Bungenberg, H. G. \& Kruyt, H. R. Procedings K. Ned. Akad. Wet. Vol. 32, 1929, pp. 849856

\footnotetext{
${ }^{*}$ Corresponding Author
} 
Camino, N. A., Sánchez, C. C., Rodríguez Patino, J. M. \& Pilosof, A. M. R. Food Hydrocolloid, Vol. 25, 2011, pp. 1-11

Carrera, C. \& Rodríguez Patino, J. M. Food Hydrocolloid. Vol. 19, 2005, pp. 407-416

Choi, S. J., Decker, E. A., Henson, L., Popplewell, L. M. \& McClements, D. J. Food Chemistry Vol. 122, 2010, pp. 111-116

Dickinson, E. An introduction to food colloids. 1992, Oxford University Press

Dickinson, E. Colloid \& Surfaces B: Biointerfaces. Vol. 20, 2001, pp. 197-210

Dickinson, E. Food Hydrocolloid, Vol. 25, 2011, pp. 1966-1983

Dickinson, E. Food Hydrocolloid. Vol. 17, 2003, pp. 25-40

Dickinson, E. Soft Matter. Vol. 4, 2008, pp. 932-942

Dmitrochenko, A.P., Antoonov, Yu. A., \& Tolseoguzov, V. B. Applied Biochemistry and Microbiology, Vol 25, 1989, 353-360

Ganzelves, R. A., Fokkink, R., van Vilet, T.,Cohen Stuart, M. A. de Jongh, H.H.J. Journal of Colloid and Interface Science Vol. 317, 2008, 137-140

Ghosh, A, \& Bandyopadhyay, P. Chemical Communications. Vol. 47, 2011, pp. 8937-8939

Grinberg, V. Y. \& Toolstoguzov, V. B. Food Hydrocolloid. Vol. 11, 1997, pp. 145-158

Jiménez-Castańo, L., Villamiel, M \& López-Fandiňo, R. Food Hydrocolloid, Vol. 21, 2007, pp. 433-443

Kabalnov, A. Journal Dispersion Science Technology Vol. 22, 2002, pp. 1-12

Koupantis, T. \& Kiosseoglou, V. Food Hydrocolloid. Vol. 23, 2009, pp. 1156-1163

Krägel, J. Derkatch, S. R. \& Miller, R. Advances in Colloid \& Interface Science. Vol. 144, 2008, 38-53

Kruif, de C. G. \& Tuinier, R. Food Hydrocolloid, Vol. 15, 2001, pp. 555-563

Kruif, de C. G., Weinbreck, F. \& Vries, de R. Current Opinion in Colloid \& Interface Science. Vol. 9, 2004, 340-349

Liu, L., Zhao, Q., Liu, T. \& Zhao, M. Food Hydrocolloid, Vol. 25, 2011, pp. 921-927

Liu, S., Low, N. H., \& Nickerson, M. T. Journal of Agricultural and Food Chemistry, Vol. 57, 2009, pp. 1521-1526

Mackie, A. Current Opinion in Colloid E Interface Science. Vol. 9, 2009, 357-361

Magnusson, E., \& Nilsson, L. Food Hydrocolloid, Vol. 25, 2011, pp. 764-772

Martínez, K. D., Sánchez, C. C., Ruíz-Henestrosa, V. P. Rodríguez Patino, J. M. \& Pilosof, A. M. R. Food Hydrocolloid, Vol. 21, 2007, pp. 813-822

McClements, D. J. Biotechnology Advances, Vol. 24, 2006, pp. 621-625

McClements, D. J. Biotechnology Advances. Vol. 24, 2006, pp. 621-625

McClements, D. J. Food Emulsions. (2 ${ }^{\text {nd }}$ Ed), 2005, Boca Raton, FL: CRC Press

McClements, D. J. Soft Matter. Vol. 7, 2011, pp. 2297-2316

McClements, D. J. Understanding and controlling the microstructure of complex foods. Abington: woodhead Publishing 2007

Mei, L. Y., Choi, S. J., Alamed, J., Henson, L., Popplewell, L. M. \& McClements, D. J. Journal of Agricultural and Food Chemistry Vol. 57, 2009, pp. 11349-11353

Mitra, R. K., Sinha, S. S. \& Pal, S. K. Langmuir, Vol. 23, 2007, pp. 10224-10229

Peniche, C., Howland, I., Carrillo, O., Zaldı'var, C., Argqelles-Monal, W., Food Hydrocoll. 18 2004, 865-871. 
Rodríguez Patino J. M., \& Pilosof A. M. R. Food Hydrocolloid, Vol. 25, 2011, pp. 1925-1937

Salgam, D., Venema, P., Vries, de R. J., Sagis, L. M. C., Linden, E. van der. Food Hydrocolloid, Vol. 25, 2011, pp. 1139-1148

Savary, G., Hucher, N., Bernadi, E., Grisel, M. \& Malhiac, C. Food Hydrocolloid, Vol. 24, 2010, pp. 178-183

Schmidt, K. A \& Smith, D. E. Journal of Dairy Science. Vol.5, 1992, pp. 36-42

Schmitt C, Kolodziejczyk E. In: Williams PA, Phillips GO, editors. Gums and stabilizers for the food industry, 15. Royal Society of Chemistry; 2010. p. 211-21

Schmitt, C. \& Turgeon, S. L. Advances in Colloid E Interface Science. Vol. 167, 2011, 63-70

Schmitt, C., Kolodziejczyk E., Lesser, M. E. Food Colloids: interactions, microstructure and processing, RSC, 2005, pp. 284-300

Schmitt, C., Sanchez, C., Desobry-Banon, S., \& Hardy, J. Critical Reviews in Food Science and Nutrition, Vol. 38, 1998, pp. 689-753

Schorsch, C., Jones, M. G. \& Norton, I. T. Food Hydrocolloid. Vol. 13, 1999, pp. 89-99

Stone, A. K., Nickerson, M. T. Food Hydrocolloid, Vol. 27, 2012, pp. 271-277

Tcholakova, S., Denkov, N. D., \& Lips, A. (2008). Physical Chemistry Chemical Physics, 10, $1608-1627$

Tolstoguzov, V. Food Polysaccharides and their Application, Taylor \& Francis, 2006, pp. 589-627

Turgeon, S. L., Schmitt, C., \& Sanchez, C. Current Opinion in Colloid \& Interface Science. Vol. $12,2007,166-178$

Weinbreck, F., Minor, M., de Kruif, C., G., J. Microencapsul., 21, 2004, 667

Weinbreck, F., Nieuwenhuijse, H., Robijn, G. W, de Kruif, C. G. Agricultural and Food Chemistry, Vol. 52, 2004, pp. 3550-3555

Xia, J, \& Dubin, P. L. Macromolecular Complexes in Chemistry and Biology, Springer-Verlag, Berlin, 1994, pp. 247

Yeo, Y., Bellas, E., Firestone, W., Langer, R., Kohane, D. S., J Agric Food Chem., 53, 2005, 7518 